\title{
Continuous haemodynamic monitoring in an unusual case of swallow induced syncope
}

\author{
Kazushi Deguchi, Christopher J Mathias
}

Autonomic Unit and University

Department of Clinical

Neurology, National

Hospital for Neurology

and Neurosurgery,

Queen Square,

Institute of Neurology,

University College

London, and

Neurovascular

Medicine Unit,

Division of

Neuroscience and

Psychological

Medicine, Imperial

College School of

Medicine at St Mary's,

London, UK

K Deguchi

C J Mathias

Correspondence to: Professor C J Mathias,

Neurovascular Medicine

Unit (Pickering Unit),

Division of Neuroscience

and Psychological Medicine,

Imperial College School of

Medicine at St Mary's, Praed

Street, London W2 1NY,

UK.

Received 20 July 1998 and in revised form

12 January 1999

Accepted 10 February

\begin{abstract}
A 69 year old man is described with a 12 year history of intermittent syncope associated with ingesting solid food, mainly after having fasted. He was taking enalapril, propranolol, bendrofluazide (bendroflumethiazide), omeprazole, finasteride, and aspirin. Detailed investigations, including gastrointestinal evaluation, measurement of various gut hormones, and autonomic testing, indicated no abnormality. A liquid meal, performed before fasting, failed to elicit an episode. However, a solid meal after an overnight fast provoked near-syncope. Continuous non-invasive haemodynamic monitoring (with a Portapres II) indicated a short lived rise in blood pressure and heart rate, followed by severe hypotension, a fall in stroke volume and cardiac output, and then bradycardia. This favoured an initial increase in sympathetic activity, followed by vasodepression due to sympathetic withdrawal or activation of humoral vasodilatatory mechanisms, with bradycardia secondary to impaired cardiac filling. Withdrawal of enalapril abolished the episodes. The unusual nature of this case, in which haemodynamic recordings continuously were made during and after swallow syncope, induced soon after food ingestion, is discussed.

(F Neurol Neurosurg Psychiatry 1999;67:220-222)
\end{abstract}

Keywords: bradycardia; hypotension; neurally mediated syncope; swallow syncope

Swallow induced or deglutition syncope is a rare disorder, characterised by loss of consciousness during or soon after a swallow. It is considered to be a form of neurally mediated syncope, with an increase in cardiac parasympathetic activity resulting in sinus bradycardia or atrioventricular block, ${ }^{1-3}$ There has been no description of continuous cardiovascular recordings with measurement of various haemodynamic parameters before, during, and after an episode. We report an unusual case in which syncope occurred soon after food ingestion.

\section{Case report}

The patient was a 69 year old man referred because of syncopal attacks that had begun 12 years previously. In a typical episode he would have paraesthesiae over the face and fingers, followed by facial flushing and redness of the eyes. The feet and hands became red and blotchy and at times he developed palpitations with a rapid but regular beat. On occasions he had visual loss and slowing of speech and then felt faint and would have to lie down. Sometimes he lost consciousness. After a syncopal attack he often felt unwell for a variable period of time; in half of these he later had profuse watery diarrhoea. The attacks usually were precipitated when he had fasted for more than 3-4 hours. Eating small amounts of food at frequent intervals reduced, but did not abolish, the attacks. These always were associated with meals and with solid, but not liquid, food. Non-syncopal attacks could occur several times a day, and the syncopal episodes occurred on at least six occasions, when fasted overnight for investigational and surgical procedures. He had heartburn suggestive of reflux oesophagitis, especially at night. He had been investigated in detail in the past. No abnormalities were seen on endoscopy, barium swallow, and abdominal ultrasound. He was taking omeprazole. In 1991, a colonic carcinoma (Duke B) was removed successfully; this did not influence the attacks. Rarer disorders with a humoral basis, such as gut hormone abnormalities and mastocytosis, were considered; a bone marrow, skin biopsy, and histamine and various gut hormone and peptide (gastrin, glucagon, vasoactive intestinal polypeptide, somatostatin, neurotensin, and pancreatic polypeptide) measurements were within normal limits. The carcinoid syndrome was excluded. There were no cardiac abnormalities. Investigations that necessitated fasting often precipitated an attack. An EEG soon after an attack was normal. Thorax CT showed an air filled oesophagus to the level of the left main bronchus. He had been hypertensive for many years and had a right internal capsular stroke from which he made a good recovery. He was started on enalapril 12 years previously.

On clinical examination the key findings were that he was overweight $(97 \mathrm{~kg})$ with a blood pressure of $170 / 90 \mathrm{~mm} \mathrm{Hg}$ (without a postural fall), a heart rate of 80 beats $/ \mathrm{min}$, and minimal reduction of fine finger and foot movements on the left, with an equivocal left plantar response. Fundoscopy showed a right 

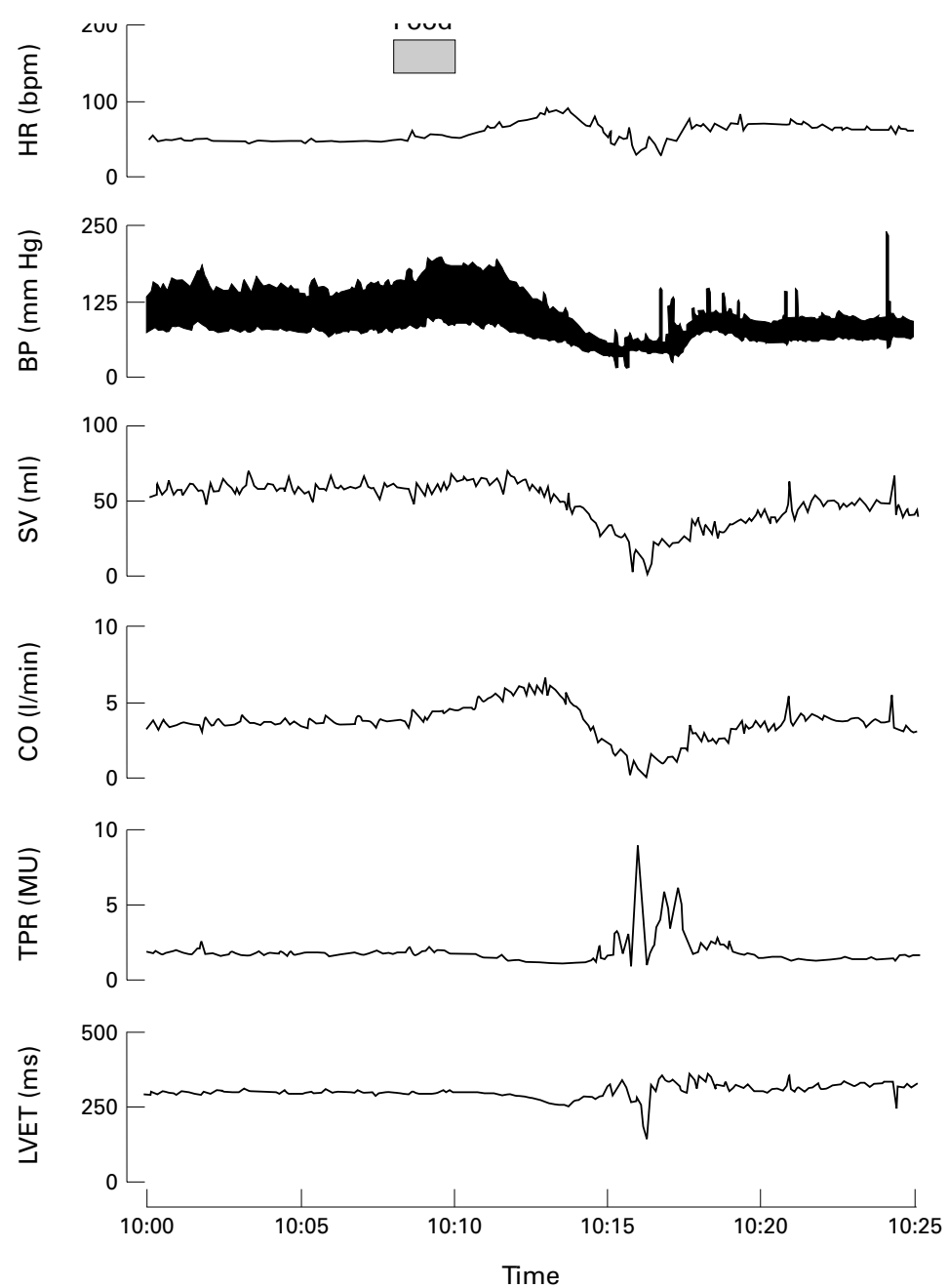

Continuous measurement of heart rate (HR), blood pressure (BP), stroke volume (SV), cardiac output (CO), total peripheral vascular resistance (TPR), and left ventricular ejection time (LVET) before, during, and after eating sandwiches, while sitting after an overnight fast. Blood pressure, heart rate, and cardiac output initially rose and then fell. Bradycardia occurred later. The irregularity in the trace occurred when the patient was near collapse and had to be laid flat. Heart rate then recovered, but stroke volume, cardiac output, and blood pressure remained low.

maculopathy that probably was age related. There were no other neurological abnormalities. A series of haematological and biochemical tests, including blood glucose concentrations, were normal. Brain CT, investigation of vestibular function, and cinevideofluroscopic examination of deglutition showed no abnormality. He underwent detailed autonomic testing, using various physiological stimuli, ${ }^{4}$ which excluded autonomic failure. $\mathrm{He}$ also underwent a liquid meal challenge, with tilt testing both before and after the meal. ${ }^{5}$ In the non-fasted state the responses were within normal limits; in the fasted state there was a fall in supine blood pressure (from 159/93 to 128/79 $\mathrm{mm} \mathrm{Hg}$ ) accompanied by facial flushing, mottling of skin over the hands, and facial sweating, from which he rapidly recovered. In view of his history he was studied after an overnight fast. Measurements on this occasion were made with a Portapres II (Biomedical TN) which provided continuous recordings of finger blood pressure, heart rate, and derived haemodynamic variables. ${ }^{6}$
On the morning of the study, premeal, the various cardiovascular autonomic measurements while sitting were stable (figure). $\mathrm{He}$ then ate egg sandwiches. There was a rise in blood pressure, cardiac output, and then heart rate with facial flushing, sweating, and bulbar conjunctival injection. This was followed after 2 minutes by a progressive fall in blood pressure, stroke volume, and then cardiac output. Heart rate continued to rise for a further 2 minutes and then fell from 108 to 58 beats/min. He had blurred vision, became confused, and was noted to have facial pallor. $\mathrm{He}$ was near collapse and was laid horizontal on his side, when he vomited clear fluid. The blood pressure slowly recovered and remained low over 48 minutes of observation.

During these investigations he had remained on his medication. In view of the association of the attacks with the initiation of enalapril, the drug was stopped. $\mathrm{He}$ has had no further attacks during a 4 year follow up period despite having to fast overnight for various investigative and surgical procedures. However, on one occasion, when enalapril was reintroduced, he had a further attack after eating breakfast but without syncope; the enalapril was stopped.

\section{Discussion}

The findings suggested that the syncopal episodes associated soon after food ingestion were neurally mediated, initially with vasodepression and then bradycardia. In swallow induced syncope the afferent pathway of the abnormal reflex is thought to involve the glossopharyngeal or vagus nerve. ${ }^{13}$ The first may be associated with glossopharyngeal neuralgia ${ }^{7}$ and the second may be due to mechanical irritation caused by oesophageal distension or spasm, or stimulation of associated structures such as the heart. ${ }^{8-11}$ In our patient the episode occurred soon after eating, suggesting that the afferent arc was in the pharynx and upper oesophagus. He did not have glossopharyngeal neuralgia. Detailed examination excluded a structural abnormality, although an air filled oesophagus was found on CT. Only solid meals, especially after fasting, induced an attack; he had symptoms suggestive of gastrooesophageal reflux, and it may be that with fasting there was increased acid reflux and also oesophageal spasm that additionally contributed. ${ }^{12}$ The precise afferent pathway, therefore, was difficult to identify.

Previous studies indicate that the efferent pathway in swallow induced syncope is likely to be the vagus, as atropine or cardiac demand pacing prevents bradycardia ${ }^{12}$; however, it may not prevent vasodepression. Abberant communication between vagal afferent and efferent fibres after trauma, demyelination, and mechanical irritation (ephaptic crosstalk), or inappropriate connections of oesophageal afferents onto medullary cardiovascular interneurons (central crosstalk) has been postulated. ${ }^{1}{ }^{12}$ In our patient the blood pressure fell before heart rate. The reasons for vasodepression, based mainly on other forms of neurally mediated syncope, ${ }^{13}$ include withdrawal of sympathetic neural activity or activation of 
vasodilatatory mechanisms. The latter include active cholinergic vasodilatation ${ }^{14}$ and the effects of local or circulatory humoral substances, such as nitric oxide ${ }^{13}$ and adrenaline (epinephrine). ${ }^{15} 16$ The initial rise in blood pressure and heart rate, with facial flushing, was consistent with adrenaline release. The subsequent fall in blood pressure, followed by a reduction in stroke volume and cardiac output, favoured vasodilatation as an initiating factor causing vasodepression. The reduction in cardiac filling then may have stimulated cardiac ventricular mechanoreceptors and a BezoldJarisch reflex, and caused bradycardia. ${ }^{17}$ After the episode, despite the heart rate returning rapidly to baseline, blood pressure, stroke volume, and cardiac output remained low, with a slow recovery. Thus, vasodilatation induced hypotension seemed to be a major initiating feature, with bradycardia playing a relatively minor part.

Syncope occurred soon after food ingestion, and therefore this differed from postprandial hypotension, as described in patients with primary autonomic failure (and postural hypotension), due to pure autonomic failure and multiple system atrophy, ${ }^{18} 19$ in whom there is sympathetic vasoconstrictor failure. There was no evidence of autonomic failure or postural hypotension on detailed autonomic testing in our patient. Furthermore, classic postprandial hypotension occurs within 15 minutes of food ingestion and reaches a nadir at about $60 \mathrm{~min}$ utes, with the low blood pressure persisting for a prolonged period, of up to 3 hours after a meal, ${ }^{18}$ presumably because of the inability of the autonomic nervous system to respond to food induced splanchnic vasodilatation. The features in our patient on liquid meal testing (and even subsequent solid meal challenge) did not favour postprandial hypotension as occurs in autonomic failure, and the episode seen was consistent with neurally mediated syncope.

Various medications can lower blood pressure ${ }^{20}$ through inappropriate reflexes. This includes angiotensin converting enzyme inhibitors, especially after the first dose, through facilitation of parasympathetic activity and the Bezold-Jarisch reflex. ${ }^{21}$ This does not occur with repeated usage. However, ACE inhibitors can cause chronic cough and the substances implicated include bradykinin, ${ }^{22}$ which may have contributed to vasodilatation through mechanisms such as sensory nerve facilitation and release of nitric oxide. Because of the possible association with the attacks, enalapril was stopped. The attacks have not recurred, except on rechallenge, despite his fasting for various investigative and operative procedures. Enalapril therefore, by mechanisms that are unclear, may have contributed to the presyncopal and syncopal episodes.

We thank Katherine Bleasdale-Barr and Maria Alam for technical assistance. Gastrointestinal, endocrine, cardiological, and Levy, Professor S Bloom, Dr J Raftery, and Dr A Bronstein.

1 Levin B, Posner JB. Swallow syncope. Report of a case and review of the literature. Neurology 1972;22:1086-93.

2 Kadisch AH, Wechsler L, Marchlinski FE. Swallowing yncope: observations in the absence of conduction system or oesophageal disease. Am f Med 1986;81:1098-100. 3 Kalloo AN, Lewis JH, Maher K, et al. Swallowing. An unu-
sual cause of syncope. Dig Dis Sci 1989;34:1117-20.

4 Mathias CJ, Bannister R. Investigation of autonomic disorders. In: CJ Mathias, R Bannister, eds. Autonomic failure. A textbook of clinical disorders of the autonomic nervous system. 4th ed. Oxford: Oxford University Press, 1999:169-95.

5 Mathias CJ, Holly E, Armstrong E, et al. The influence of food on postural hypotension in three groups with chronic autonomic failure: clinical and therapeutic implications. $f$ Neurol Neurosurg Psychiatry 1991;54:726-30.

6 Veerman DP, Imhorz BP, Wieling W, et al. Circadian profile of systemic hemodynamics. Hypertension 1995;26:55-9.

7 Odeh M, Oliven A. Glossopharyngeal neuralgia associated with cardiac syncope and weight loss. Arch Otolaryngol with cardiac syncope and weight
Head Neck Surg 1994;120:1283-6.

Head Neck Surg 1994;120:1283-6.
8 Wik B, Hillestad L. Deglutive syncope. BMF 1975;ii: 747.

9 Bortolotti M, Cirignotta F, Labo G. Atrioventricular block induced by swallowing in a patient with diffuse esophageal spasm. ҒAMA 1982;248:2297-9.

10 Nakagawa S, Hisanaga S, Kondoh H, et al. A case of swallow syncope induced by vagotonic visceral reflex resulting in atrioventricular node suppression. F Electrocardiol 1987;20: $65-9$

11 Bortolotti M, Sarti P, Brunelli F, et al. Abnormal esophagocardiac inhibitory reflex in patients with diffuse esophageal spasm. Digestion 1995;56:488-92.

12 Cunningham ET, Ravich WJ, Jones B, et al. Vagal reflexes referred from the upper aerodigestive tract: an infrequently recognised cause of common cardiorespiratory response. recognised cause of common
Ann Int Med 1992;116:575-82.

13 Kaufmann H. Neurally mediated syncope: pathogenesis, diagnosis and treatment. Neurology 1995;45(suppl 5):S1218

14 Saunders JC, Mark AL, Fergusson DW. Evidence for cholinergically mediated vasodilatation at the beginning of exercise in humans. Circulation 1989;79:815-24.

15 Robinson BJ, Johnson RH. Why does vasodilatation occur during syncope? Clin Sci 1988;74:347-50.

16 Smith GDP, Watson LP, Mathias CJ. Cardiovascular and catecholamine changes induced by supine exercise and upright posture in vasovagal syncope. Comparisons with normal subjects and subjects with sympathetic denervation. Eur Heart f 1996;17:1882-90.

17 Hainsworth R. Syncope and fainting. In: CJ Mathias, R Bannister, eds. Autonomic failure. A textbook of clinical disorders of the autonomic nervous system. 4 th ed. Oxford: Oxford University Press, 1999:428-36.

18 Mathias CJ, da Costa DF, Fosbraey P, et al. Cardiovascular, biochemical and hormonal changes during food induced hypotension in chronic autonomic failure. 7 Neurol Sci 1989;94:255-69.

19 Mathias CJ, Bannister R. Postcibal hypotension in autonomic disorders. Mathias CJ, Bannister R, eds. Autonomic failure: a textbook of clinical disorders of the autonomic nervous system. 4th ed. Oxford: Oxford University Press, 1999:28395.

20 Hanlon JT, Linzer M, MacMillan JP, et al. Syncope and presyncope associated with probable adverse drug reactions. Arch Intern Med 1990;150:2309-12.

21 Reid JL, Millar JA, Campbell BC. Enalapril and autonomic reflexes and exercise performance. $\mathscr{f}$ Hypertens 1983; 1(suppl 1):S129-34.

22 Yeo WW, Chadwick IG, Kraskiewicz M, et al. Resolution of ACE inhibitor cough: changes in subjective cough and
responses to inhaled capsaicin, intradermal bradykinin and rubstance-P. Br f Clin Pharmacol 1995;40:423-9. 